\title{
Overexpression of ECM1 Contributes to Migration and Invasion in Cholangiocarcinoma Cell
}

\author{
G. P. XIONG ${ }^{1,2}$, J. X. ZHANG'2, S. P. GU³, Y. B. WU', J. F. LIU ${ }^{4, *}$ \\ ${ }^{1}$ Post graduate college, Fujian medical university, FuZhou 350004, China; ${ }^{2}$ Department of hepatobiliary surgery, Liver Disease Center of Xiamen \\ Traditional Hospital, Xiamen 361000, Fujian, China; ${ }^{3}$ Minimally invasive surgery department of shishi city hospital, Fujian, 362700, China; \\ ${ }^{4}$ Department of Hepatic surgery, Liver Disease Center of the First Affiliated Hospital of Fujian Medical University, Fuzhou 350005, China \\ ${ }^{\star}$ Correspondence: jingfengliu@126.com
}

Received October 31, 2011 / Accepted February 3, 2012

\begin{abstract}
Although the expression of extracellular matrix protein-1 (ECM1) has been documented in several tumor models, the function of ECM1 has remained unclear. In this study, expression of ECM1 was detected by real time PCR and immunohistochemistry. The role and mechanism of ECM1 overexpression in cholangiocarcinoma (CCA) cells were assessed by wound-healing, matrigel invasion assay and Western blotting. Expression of ECM1 was significantly elevated in CCA tissues than that in adjacent noncancerous, cholangitis and normal bile duct tissues. Its overexpression was associated with poor differentiation, lymph node metastasis, poor prognosis, and the level of CA199, MMP-9, estrogen receptor. Knockdown of ECM1 suppressed migration and invasion of CCA cells. Using PI3K or IKK inhibitor reduced the level of phospho-Akt or phospho-IкBa as well as ECM1. Taken together, overexpression of ECM1 may contribute to CCA initiation and progression through promoting migration and invasion of CCA cells, its overexpression was associated with Akt/NF- $\mathrm{kB}$ signaling axis.
\end{abstract}

Key words: extracellular matrix protein 1, migration, invasion, Akt/NF- $\kappa B$, cholangiocarcinoma

The morbidity and mortality rates of cholangiocarcinoma (CCA) have increased rapidly in past three decades in Northeastern Thailand, China and Japan [1]. As a result of the early invasion and metastasis, majority of CCA patients are precluded from curative surgical resection and have a short-term survival [2]. Thus, novel predictors of invasive phenotypes of CCA are critically needed.

Indeed, CCA has an early propensity to metastasize to regional lymph nodes, the peritoneum, and the omentum [2-3]. Traditionally, the growth and metastasis of tumor cells depend on the support of the extracellular matrix (ECM) milieu. It is now widely accepted that the exchange of information between the ECM and tumor cells is essential to cellular interaction and tumorigenesis [4]. However, our knowledge of these complex interactions remains incomplete. The extracellular matrix protein-1 (ECM1) was originally identified as an $85-\mathrm{kDa}$ glycoprotein secreted by the murine osteogenic stromal cell line MN7 [5]. Until now, the function of ECM1 has remained unclear. Its expression has been documented in several tumor models and it may play a role in carcinogenesis by promoting angiogenesis, invasion and metastasis in these cancer models [6-10]. At present, little is known about the role of ECM1 in CCA.

The aim of this study was to investigate the expression and clinical significance of ECM1 in a hospital-based cohort of CCA patients and to determine the role of ECM1 in the development of CCA and the mechanism of its overexpression.

\section{Patients and Methods}

Patients and tumor specimens. Fresh surgical specimens of both CCA and the adjacent noncancerous tissues (44), cholangitis (17) and normal bile duct tissues (12) were obtained from 2007 to 2010 in the Department of Hepatobiliary Surgery, Xiamen Hospital of Traditional Chinese Medical, which was approved by the institution's Human Investigation Committee. None of patients had received preoperative treatment and metastatic tumors from other tissue origins were excluded from this study. All patients gave written informed 
consent. The clinicopathologic data of CCA patients were listed in Table 1.

Follow-up was made by out-patient clinic interview, telephone or letter communication. The follow-up period was 1 years to 3 years (median 1.2 years).

Reagents. Lipofectamine2000, Lipofectamine ${ }^{\mathrm{TM}}$ RNAiMAX, Stealth-siRNA (Invitrogen); LY-294002 and BMS-345541, SB239063, Nimesulide, PD98059 (Sigma); Polyclonal antibodies against ECM1, Akt, p-Akt (Abcom), and monoclonal antibodies against MMP-2, MMP-9, ER, Her-2, $\beta$-actin, IкB $\alpha$, p-IкBa (Santa Cruz Biotechnology); Goat anti-rabbit/mouse secondary antibodies, enhanced chemilumienescence (ECL) reagents (Tiangen Biotech Co); Polyvinylidene difluoride (PVDF) membranes (Millipore). Matrigel Invasion Chambers (BD Biosciences).

Cell cultures and transfection. Human CCA cell lines QBC939 and SK-Cha-1 were cultured in RPMI-1640 medium supplemented with $10 \% \mathrm{FBS}, 100 \mathrm{U} / \mathrm{ml}$ penicillin at $37^{\circ} \mathrm{C}$ in an atmosphere of air containing $5 \% \mathrm{CO}_{2}$. Target stealth-siRNA (ECM1: GAGGATACCCTTGACAAATACTGTG) and nonspecific stealth-siRNA (GCAATGCCAGTATCAACGTTGGAAT) were transfected with Lipofectamine ${ }^{\mathrm{TM}}$ RNAiMAX according to manufacture's instructions. To generate stably transfected cells, CCA cells were respectively transfected

Table1. Relationships between ECM1 and CCA

\begin{tabular}{|c|c|c|c|c|}
\hline \multirow[b]{2}{*}{ Fetures } & \multirow[b]{2}{*}{$\mathbf{N}$} & \multicolumn{3}{|c|}{ ECM1 } \\
\hline & & Low & High & $P$ \\
\hline \multicolumn{5}{|l|}{ Age } \\
\hline$<50$ & 12 & 3 & 9 & 0.836 \\
\hline$\geq 50$ & 32 & 9 & 23 & \\
\hline \multicolumn{5}{|l|}{ Sex } \\
\hline Male & 29 & 11 & 18 & $0.027^{*}$ \\
\hline Female & 15 & 1 & 14 & \\
\hline Tumor differentiation ${ }^{f}$ & & & & $0.035^{*}$ \\
\hline $\mathrm{W}+\mathrm{WM}$ & 7 & 4 & 3 & \\
\hline M & 17 & 6 & 11 & \\
\hline $\mathrm{MP}+\mathrm{P}$ & 20 & 2 & 18 & 0.736 \\
\hline \multicolumn{5}{|l|}{ Tumor location } \\
\hline Intrahepatic & 13 & 4 & 9 & \\
\hline Extrahepatic & 31 & 8 & 23 & 0.419 \\
\hline \multicolumn{5}{|l|}{ Gallstone } \\
\hline Yes & 19 & 4 & 15 & \\
\hline No & 25 & 8 & 17 & $0.019^{*}$ \\
\hline \multicolumn{5}{|l|}{ Lymph node metastasis } \\
\hline Positve & 27 & 4 & 23 & 0.343 \\
\hline Negative & 17 & 8 & 9 & \\
\hline \multicolumn{5}{|l|}{ Portal invasion } \\
\hline Positve & 17 & 6 & 11 & 0.516 \\
\hline Negative & 27 & 6 & 21 & \\
\hline \multicolumn{5}{|l|}{ Microvascular invasion } \\
\hline Positve & 15 & 5 & 10 & \\
\hline Negative & 29 & 7 & 22 & \\
\hline
\end{tabular}

${ }^{\star} P$ value $<0.05$ is considered statistically significant. ${ }^{\circ} \mathrm{W}$, well differentiated; $\mathrm{WM}$, well-to-moderately differentiated. M, moderately differentiated; MP, moderately to poorly differentiated; P, poorly differentiated. with pll3.7 control vector (shCtrl) and pll3.7-ECM1 vector (shECM1) by Lipofectamine2000.

Immunohistochemistry (IHC). The paraffin-embedded sections were deparaffinized in xylene and rehydrated through graded alcohol solutions. For epitope retrieval, slides were incubated in $0.01 \mathrm{M}$ citric acid buffer ( $\mathrm{pH} 6.0$ ) at $95{ }^{\circ} \mathrm{C}$ for $20 \mathrm{~min}$. Sections were then incubated and stained according to the manufacture's instructions (ECM1: 1:300; MMP-2, MMP-9, ER, Her-2, 1:200). The negative controls were processed in similar manner with PBS instead of primary antibody. The results were expressed as percentage of cells staining on a graduated percentage $(0-100 \%)$. Staining $<10 \%$ was classified as -, $10 \%$ to $30 \%$ as,$+ 30 \%$ to $60 \%$ as ++, $60 \%$ to $100 \%$ as +++. Ten fields were selected and expression in 1,000 tumor cells (100 cells/field) was evaluated using high power microscope $(\times 400)$. Expression of proteins were categorized as low $(-$ to +$)$ or high $(++$ to +++$)[10]$. The IHC results were scored by three experienced pathologists, who were blinded to clinical data.

Western blotting. Equal amount of proteins was electrophoresed on an 10\% SDS-PAGE gel and transferred onto PVDF membranes. Following probing with a specific primary Ab (ECM1, Akt, p-Akt, 1:2000; $\beta$-actin, IкB $\alpha$, p-IкB $\alpha$, $1: 1000)$ and horseradish peroxidase-conjugated secondary $\mathrm{Ab}(1: 10000)$, the protein bands were detected by ECL.

Real-time RT-PCR. Total RNA was extracted from cells or human tissue using TRIzol Reagent and was reverse transcribed into cDNA. Quantification of the cDNA template was performed by real-time PCR using SYBR green. The primers for ECM1 were forward primer: ATGAGTGCTTTGCCCCGTCGG, reverse primer: GTTCTG GAAATGGCAGGTCAC. GAPDH, forward primer: GGAAGGTGAAGGTCGGA

GT CA, reverse primer: GTCATTGATGGCAACAATATCCACT. All primers were custom synthesized by Invitrogen Corporation.

Wound healing and matrigel invasion assays. Cells (siECM1 or siCtrl) were cultured on glass coverslips in 6-well plates. The confluent monolayers were scraped in a line across the slides with a sterile $20-\mu \mathrm{l}$ plastic pipette tip and incubated in serum-free medium for $48 \mathrm{~h}$. The cell migration was evaluated by comparing the remaining cell-free area with that of the initial scrape line. Cell invasion was determined using Matrigel Invasion Chambers. A $500 \mu \mathrm{L}$ aliquot $\left(5 \times 10^{4}\right)$ of cells (shECM1 or shCtrl) in $0.2 \%$ FBS medium was added to the upper compartment of the Transwell, and 10\% FBS medium was added to the lower chamber. After $48 \mathrm{~h}$ of incubation at $37^{\circ} \mathrm{C}$ in a humidified $\mathrm{CO}_{2}$ incubator, non-invaded cells in the upper compartment were removed with a cotton swab, and the invaded cells were stained and counted.

Statistical analysis. All data were processed with SPSS 16.0 statistical software package. The Pearson's $\chi^{2}$ were used to analyse the data from IHC. Overall survival of patients were estimated by Kaplan-Meier method. Other data were expressed as mean \pm SD and analyzed by Student's $t$ test. Each assay was 
repeated in triplicate in three independent experiments. Value of $P<0.05$ was considered significant.

\section{Results}

Expression of ECM1 is elevated in CCA. To determine the expression of ECM1 in CCA, we first measured its mRNA expression by real time PCR in both CCA and their adjacent noncancerous tissues, as well as cholangitis and normal bile duct tissues. The results showed ECM1 mRNA level was significantly elevated in CCA tissues (Figure 1A). Furthermore, we detected the protein level of ECM1 by IHC which showed a predominantly, although not exclusively, cytoplasmic staining, and was not uniformly high in CCA tissues for ECM1 (Figure 1B). However, the expression of ECM1 protein was significantly elevated in CCA tissues (72.7\%) compared with their adjacent noncancerous tissues $(40.9 \%)$, cholangitis (41.2\%) or normal bile duct (33.3\%) tissues, but there was no significant difference of ECM1 expression among noncancer tissues, cholangitis and normal bile duct tissues (Table 2). Together, these results suggest that the expression of ECM1 is significantly elevated in CCA tissues.

Overexpression of ECM1 is associated with aggressive behaviours of CCA. Overexpression of ECM1 in CCA may contribute to the development of CCA, so we evaluated the associations between ECM1 and clinicopathological characteristics. The results from Table 1 showed that overexpression of ECM1 was significantly associated with patient sex, poor differentiation of CCA (Figure 2A), and lymph node metastasis (Figure 2B). No association was found between ECM1 and patient age, tumor location, gallstone, portal invasion, microvascular invasion. How did the ECM1 overexpression contribute to prognosis? Kaplan-Meier method was performed to assess the effect of ECM1 overexpression on overall survival. As shown in Figure $2 \mathrm{C}$, overall survival rate after surgical resection was significantly worse in patients who had tumors exhibiting ECM1 high expression (median survival time, 7.9 months; cumulative 3 -year survival rate, $0 \%$ ) than in patients who had tumors that were low of ECM1 expression (median survival time, 32.5 months; cumulative 3-year survival rate, $21 \% ; P=0.005)$. Furthermore, we also assessed the relationships between ECM1 overexpression and the indexes associated with CCA prognosis. The results from Table 3 showed that overexpression of ECM1 was significantly associated with CA199, MMP-9 and estrogen receptor. Taken together, these results suggest that overexpression of ECM1 may contribute to CCA initiation and progression.

Overexpression of ECM1 contributes to migration and invasion of CCA cells. In order to address the functional role of ECM1 in CCA cells, we tried to inhibit ECM1 expression by siRNA and tested the effect of siRNA on the proliferation of CCA cells. However, we found that downregulation of ECM1 did not affect cell proliferation (data not showed). Previous reports showed that ECM1 was associated with metastasis in several cancer cells $[4,6,10]$. We then used the wound-healing
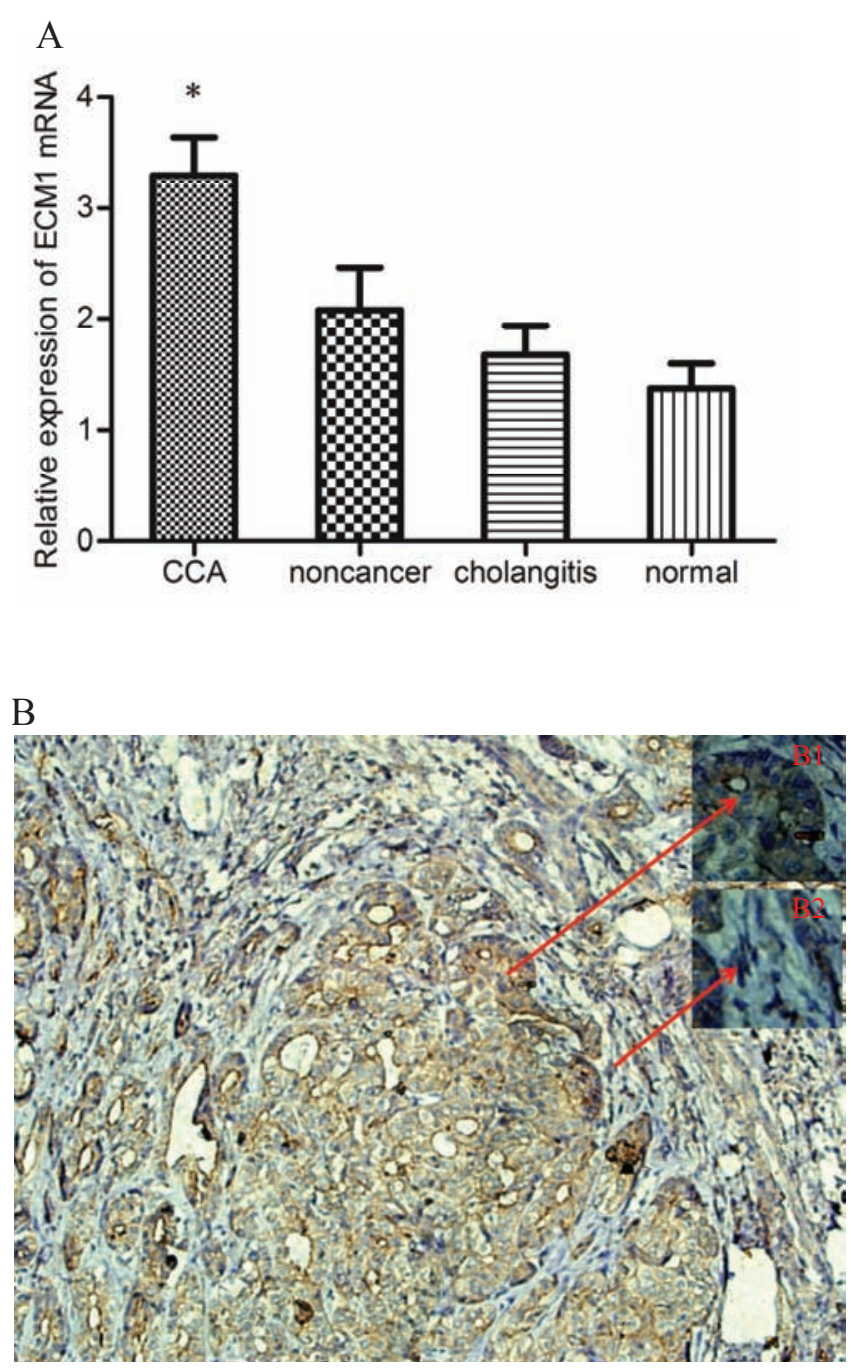

Figure 1. Overexpression of ECM1 in CCA tissues. (A) Total RNA samples were extracted from CCA, adjacent noncancer, cholangitis, and normal bile duct tissues. Expression of ECM1 mRNA was assessed by real-time RT-PCR. Data were normalized to GAPDH and were expressed as ratios of ECM1/GAPDH $\times 10^{3} . p$ value less than 0.05 is considered statistically significant, which are denoted with "*”. (B) Human CCAs and their adjacent noncancer tissues were immunostained with antibody against ECM1. As the arrows designate, ECM1 highly expressed in the cytoplasm of CCA cells (B1), but low or no stained in adjacent noncancer cells (B2).

Table 2. Expession of ECM1 in different bile duct tissues

\begin{tabular}{lrrr}
\hline & \multicolumn{3}{c}{ ECM1 } \\
\cline { 2 - 3 } Tissues & Low & High & $P$ \\
\hline CCA $^{1}$ & 12 & 32 & 1 vs. 2 vs. 3 vs. 4: 0.005 \\
Noncancer $^{2}$ & 26 & 18 & \\
Cholangitis $^{3}$ & 10 & 7 & 2 vs. 3 vs. 4: 0.883 \\
Normal $^{4}$ & 8 & 4 & \\
\hline
\end{tabular}

Vs: Statistical analysis by chi-square test, $P<0.05$ is considered statistically significant. 


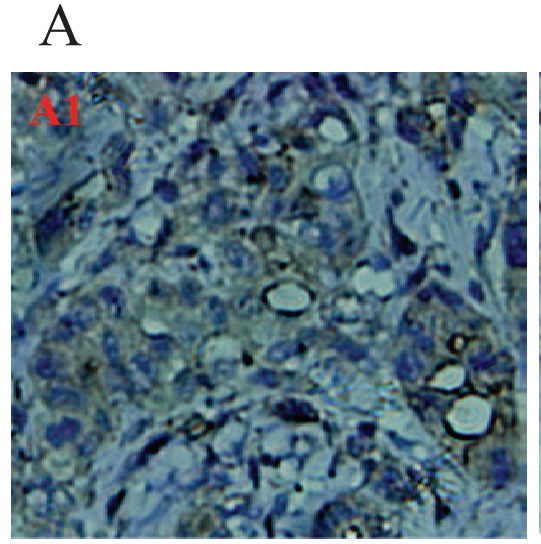

Well differentiated

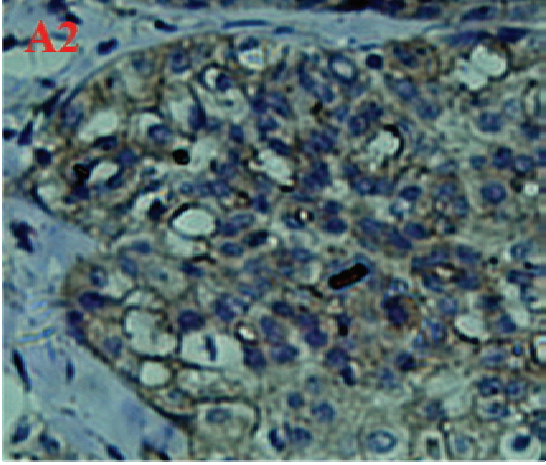

Moderately differentiated

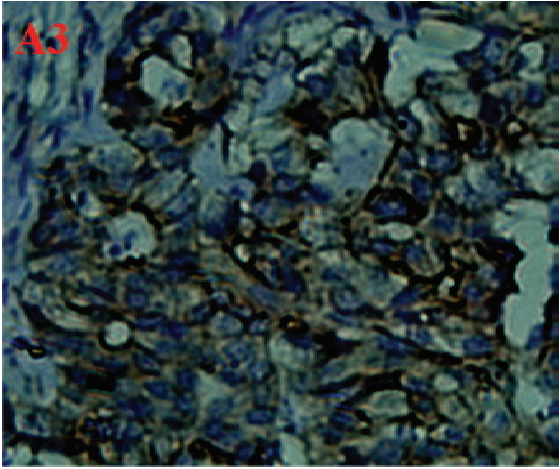

Poorly differentiated $(200 \times)$

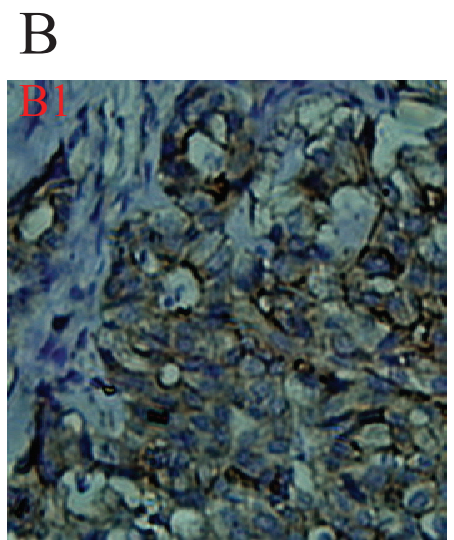

Metastasis

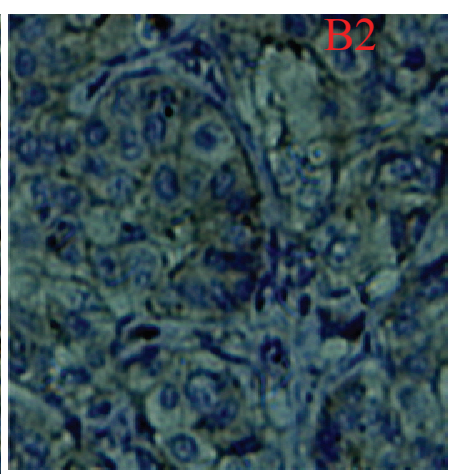

Non-metastasis

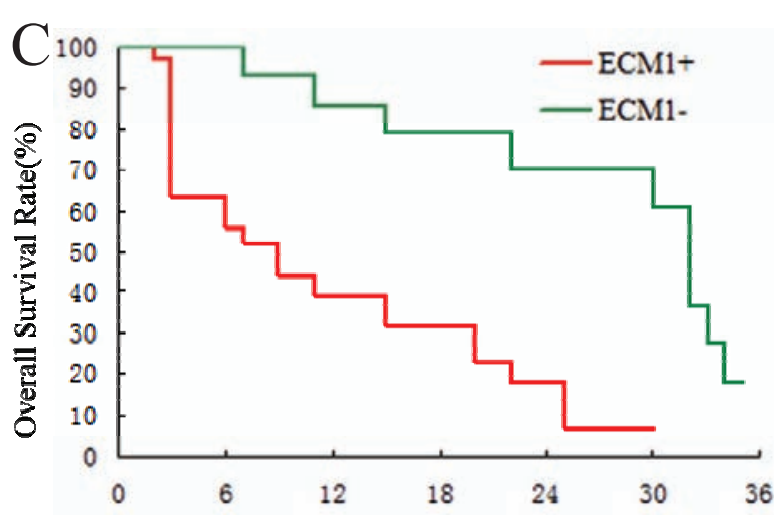

Months after Surgery (m)

Figure 2. Association of ECM1 with clinicopathological characteristics of CCA.

(A) Immunohistochemical staining of ECM1 in different CCA differentiation tissues. A1, + ECM1staining in well differentiated; A2, ++ ECM1staining in moderately differentiated; A3, +++ ECM1staining in poorly differentiated. (B) Note that more cells in metastasis tumor exhibited strong nuclear staining for ECM1 (B1), but weak staining in non-metastasis tumor tissues (B2). (C) Kaplan-Meier curve showing the effects of ECM1 overexpression on overall survival.

assay to investigate the effect of ECM1 siRNA in migration of CCA cells. As showed in Figure 3A, downregulation of ECM1 decreased cell migration rate in SK-ChA-1, compared to control siRNA. Stable downregulation of ECM1 in shECM1 transfected CCA cells was constructed to confirm this data. Matrigel invasion assay was used to assess the migration and invasion of shECM1 and shCtrl cells. The results in Figure 3B showed that migration and invasion of SK-ChA-1 cells were significantly reduced when ECM1 was knockdowned, and a similar results were observed in QBC939 cells. These results suggest that overexpression of ECM1 plays an important role in migration and invasion of CCA cells.

Activation of Akt/NF-kB signaling axis contributes to ECM1 overexpression.

In order to determine the potential mechanism of ECM1 overexpression in CCA, we chose several signaling pathways that regulate migration and invasion of CCA cells including
COX-2/PGE2, PI3K/ AKT, ERK1/2, and p38 [11-13]. We examined the effects of those signaling pathways inhibition (by their inhibitors) on the expression of ECM1. Eventually, we found that suppression of PI3K/ AKT by LY294002 (48h) reduced level of phospho-AKT and ECM1, but without affecting total AKT level (Figure 4A). We next studied whether activation of NF- $\kappa \mathrm{B}$, a general downstream target of PI3K/ $\mathrm{AKT}$, was required for overexpression of ECM1. As showed in figure 4B, IKK inhibitor BMS-345541 could reduce the level of phospho-IкB $\alpha$ and ECM1. Taken together, the results suggest that activation of Akt/NF- $\kappa B$ signaling axis may contribute to ECM1 overexpression.

\section{Discussion}

Our results showed that the level of ECM1 mRNA and protein were significantly elevated in a majority of CCA tis- 
A

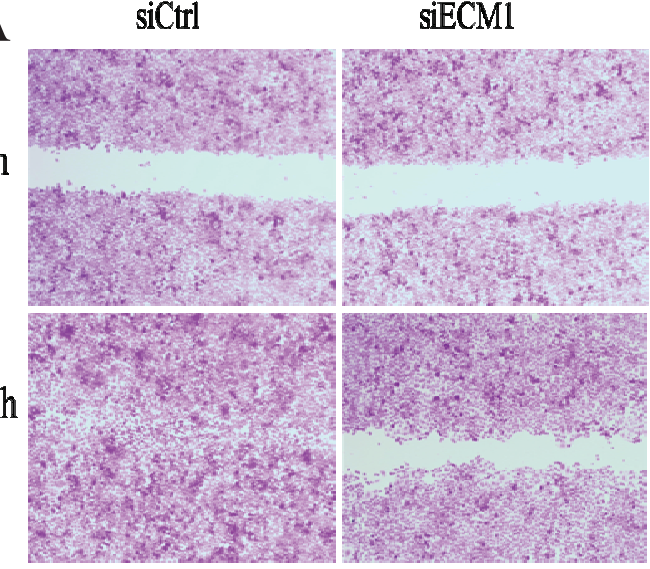

siCtrl
$0 \mathrm{~h}$

$48 \mathrm{~h}$

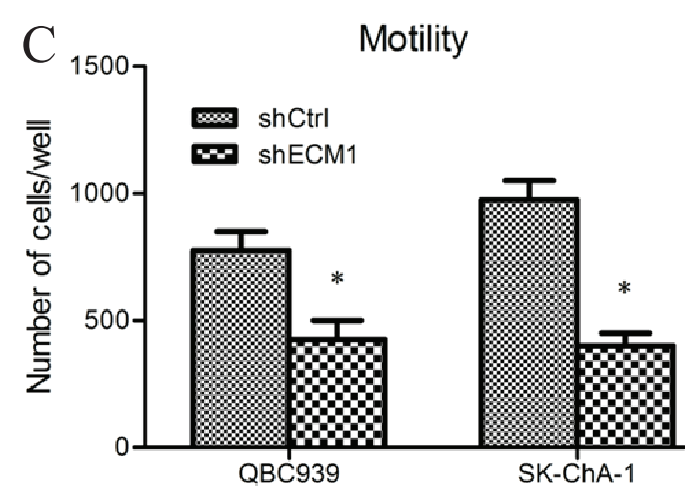

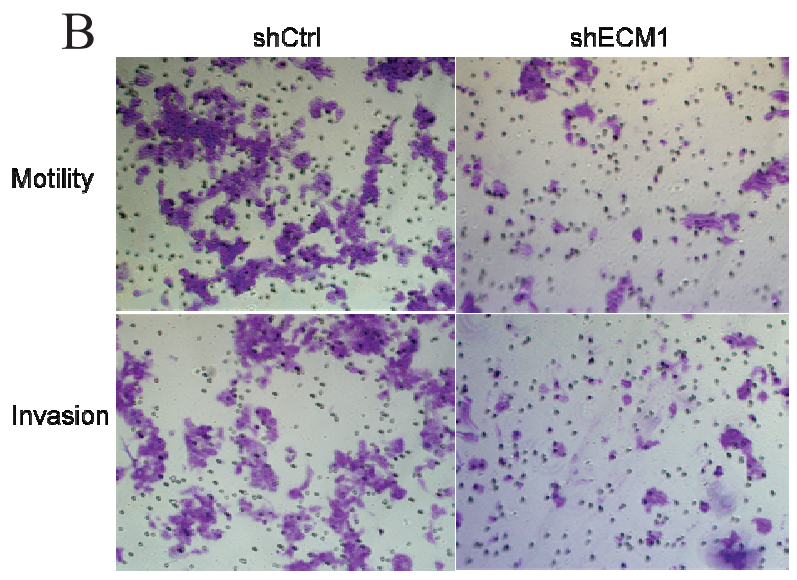

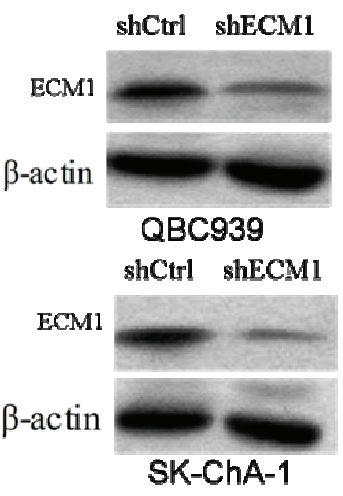

Figure 3. Effect of ECM1 knockdown on motility and invasion of the CCA cells.

(A) Downregulation of ECM1 in SK-ChA-1 cells reduced migration in wound healing assay. The confluent monolayers of CCA cells were scratched and allowed to migrate up to $48 \mathrm{~h}$. The remaining cell-free area in siCtrl is less than siECM1. (B) Knockdown of ECM1 in SK-ChA-1 cells reduced migration and invasion in matrigel invasion assay. Equal cells for shCtrl and shECM1 were allowed to invade through the matrigel up to 60h or migrate through membrane without matrigel up to $48 \mathrm{~h}$. Invaded cells were stained with crystal violet and counted to quantify. Migration and invasion figures of QBC939 were not shown. (C) Data from (B) are expressed as percent change (means \pm SEM) compared to control. Western blot analysis of ECM1 expression in shECM1 and shCtrl cells. ${ }^{*} P<0.05$.

A

LY294002
ECM1
p-Akt

Akt

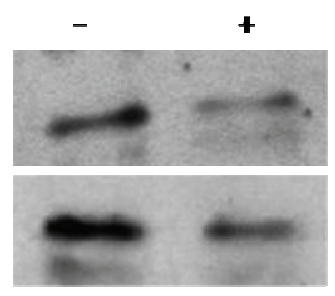

$\beta$-actin
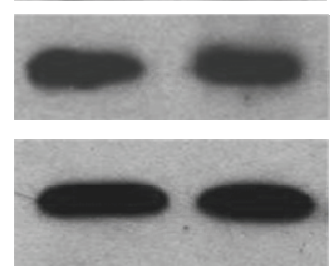

B BMS-345541 - $\quad+$

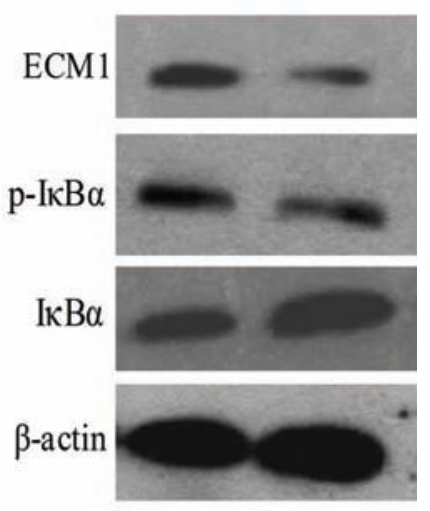

Figure 4. Effect of Akt/NF-kB signaling axis on the ECM1 expression. The SK-ChA-1 cells were treated with or without the PI3K inhibitor LY294002 $(15 \mu \mathrm{mol} / \mathrm{L})(\mathrm{A})$ or IKK inhibitor BMS-345541 (15 $\mu \mathrm{mol} / \mathrm{L})$ (B) for 48h. Phosphorylated and total levels of both Akt and IkBa were analyzed by Western blotting. The results shown are representatives of three separate experiment. 
Table 3. Relationships between ECM1 and factors affecting CCA prognosis

\begin{tabular}{|c|c|c|c|c|}
\hline \multirow{2}{*}{ Fetures } & \multirow{2}{*}{$\mathbf{N}$} & \multicolumn{3}{|c|}{ ECM1 } \\
\hline & & Low & High & $P$ \\
\hline \multicolumn{5}{|l|}{$\overline{\text { CEA }(u g / L)^{f}}$} \\
\hline$<15$ & 12 & 2 & 10 & 0.461 \\
\hline$\geq 15$ & 32 & 10 & 22 & \\
\hline \multicolumn{5}{|c|}{$\mathrm{CA} 199(\mathrm{U} / \mathrm{ml})^{+}$} \\
\hline$<37$ & 5 & 4 & 1 & $0.005^{*}$ \\
\hline$\geq 37$ & 39 & 8 & 31 & \\
\hline \multicolumn{5}{|l|}{ MMP-9 } \\
\hline Positve & 30 & 4 & 26 & $0.002^{*}$ \\
\hline Negative & 14 & 8 & 6 & \\
\hline \multicolumn{5}{|l|}{ MMP-2 } \\
\hline Positve & 20 & 5 & 15 & 1.000 \\
\hline Negative & 24 & 7 & 17 & \\
\hline \multicolumn{5}{|c|}{ Estrogen receptor } \\
\hline Positve & 33 & 5 & 28 & $0.002^{*}$ \\
\hline Negative & 11 & 7 & 4 & \\
\hline \multicolumn{5}{|l|}{ Her- $2 /$ neu } \\
\hline Positve & 20 & 4 & 16 & 0.498 \\
\hline Negative & 24 & 8 & 16 & \\
\hline
\end{tabular}

${ }^{*} P$ value $<0.05$ is considered statistically significant.

The threshold of CEA and CA199 were refered to reference 14 .

sues. We showed that overexpression of ECM1 was associated with the patient sex, poor differentiation of CCA, and lymph node metastasis, as well as several indexes of CCA prognosis CA199, MMP-9 and estrogen receptor [14-15]. There was a general inverse trend between a reduction in patient survival and increasing ECM1 staining intensity, which indicated that patients with high expression of ECM1 had significantly poorer survival. Therefore, it is proposed that overexpression of ECM1 may contribute to CCA initiation and progression.

ECM1 exerts its carcinogenic activity in several tumor models through promoting angiogenesis, invasion and metastasis in several cancer models [6,10]. Its metastatic-promoting properties have been suggested by its ability to stimulate the formation of blood vessels in the chorioallantoic membrane of chicken eggs [16], and its interaction with various skin extracellular matrix proteins, such as the heparin sulphate proteoglycan perlecan, fibulin-1C/D, MMP-9, fibronectin, laminin 332 and collagen type IV [17-18]. Data presented here show that downregulation of ECM1 expression, didn't affect the proliferation of CCA cells, suppressed the migration and invasion of CCA cells. This is consistent with the result from the clinical data that lymph-node metastases are more lilely to be ECM1-positive than that without metastases, although overexpression of ECM1 was not associated with portal invasion and microvascular invasion (maybe due to small sample size). Thus, concluding from previous studies and our investigation, we suggest that overexpression of ECM1 contributed to CCA initiation and progression through promoting the migration and invasion of CCA cells.

Although previous studies have identified that ECM1 was elevated in several human tumors, the exact mechanisms that regulates ECM1 overexpression are still unclear. Previous reports showed that ECM1 was induced by overexpression of AP2alpha in breast cancer and activation of wnt-1/betacatenin signaling in mouse mammary epithelial cells [19-20]. However, data in our study showed that suppressed PI3K/ AKT pathway or NF- $\kappa B$ signaling pathway by their specific inhibitors reduced ECM1 expression. Previous study reported that activated PI3K/AKT pathway is associated with high proliferation index, lymph node metastasis and vascular invasion, advanced tumor stage and poor prognosis in CCA, and NF- $\kappa \mathrm{B}$ is often its general downstream single pathway [12], so we propose that overexpression of ECM1 in CCA cells maybe associated with activation of Akt/NF- $\mathrm{KB}$ signaling axis.

In summary, our results highlights the crucial role of ECM1 in the development of human CCA by promoting the cell migration and invasion, and its overexpression maybe associated with activation of Akt/NF- $\mathrm{kB}$ signaling axis. Our interesting findings suggest that ECM1 may represent a therapeutic target for this devastating malignancy.

Acknowledgments: We thank all colleagues in the department of Hepatobiliary Surgery of Xiamen Hospital of Traditional Chinese Medical for collecting all kinds of bile duct tissues and the clinicopathological and follow-up data.

\section{References}

[1] ALJIFFRY M, WALSH MJ, MOLINARI M. Advances in diagnosis, treatment and palliation of cholangiocarcinoma: 1990-2009. World J Gastroenterol. 2009; 15: 4240-4262. http: //dx.doi.org/10.3748/wjg.15.4240

[2] SHEN FZ, ZHANG BY, FENG YJ, JIA ZX, AN B, et al. Current research in perineural invasion of cholangiocarcinoma. Journal of Experimental \& Clinical Cancer Research. 2010; 29: 24.

[3] HASSID VJ, ORLANDO FA, AWAD ZT, TAN D, KHOURY $\mathrm{T}$, et al. Genetic and molecular abnormalities in cholangiocarcinogenesis. Anticancer Res. 2009; 29: 1151-6.

[4] HAN Z, LIN GJ, CHI FL, WANG SY, HUANG JM, et al. The relationship between the extracellular matrix and the angiogenesis and metastasis of laryngeal carcinoma. ORL J Otorhinolaryngol Relat Spec. 2008; 70: 352-8. http: //dx.doi. org/10.1159/000163030

[5] MATHIEU E, MEHEUS L, RAYMACKERS J, MERREGAETT J: Characterization of the stromal osteogenic cell line MN7: identification of secreted MN7 proteins using two-dimensional polyacrylamide gel electrophoresis, Western blotting and microsequencing. J Bone Miner Res. 1994; 9: 903-913. http: //dx.doi.org/10.1002/jbmr.5650090616

[6] HAN Z, NI J, SMITS P, UNDERHILL CB, XIE B, et al. Extracellular matrix protein 1 (ECM1) has angiogenic properties and is expressed by breast tumor cells. FASEB J. 2001; 15: 988-94. http: //dx.doi.org/10.1096/f.99-0934com

[7] WANG L, YU J, NI J, XU XM, WANG J, et al. Extracellular matrix protein 1 (ECM1) is over-expressed in malignant epi- 
thelial tumors. Cancer Lett. 2003; 200: 57-67. http: //dx.doi. org/10.1016/S0304-3835(03)00350-1

[8] KEBEBEW E, PENG M, REIFF E, DUH QY, CLARK OH, et al. ECM1 and TMPRSS4 are diagnostic markers of malignant thyroid neoplasms and improve the accuracy of fine needle aspiration biopsy. Ann Surg. 2005; 242: 353-61.

[9] LAL G, HASHIMI S, SMITH BJ, LYNCH CF, ZHANG L, et al. Extracellular Matrix 1(ECM1) expression is a novel prognostic marker for poor long-term survival in breast cancer: a hospital-based cohort study in Iowa[J]. Ann Surg Oncol, 2009; 16: 2280-87. http: //dx.doi.org/10.1245/s10434-0090533-2

[10] CHEN H, JIA WD, LI JS, WANG W, XU GL, et al. Extracellular matrix protein 1 , a novel prognostic factor, is associated with metastatic potential of hepatocellular carcinoma. Med Oncol. 2010 Dec 3. [Epub ahead of print]

[11] ITATSU K, SASAKI M, YAMAGUCHI J, OHIRA S, ISHIKAWA A, et al. Cyclooxygenase-2 Is Involved in the Up-Regulation of Matrix Metalloproteinase-9 in Cholangiocarcinoma Induced by Tumor Necrosis Factor- $\alpha$. The American Journal of Pathology. 2009; 174: 829-841. http: //dx.doi.org/10.2353/ajpath.2009.080012

[12] MENAKONGKA A, SUTHIPHONGCHAI T. Involvement of PI3K and ERK1/2 pathways in hepatocyte growth factor-induced cholangiocarcinoma cell invasion. World J Gastroenterol. 2010; 16: 713-722. http://dx.doi.org/10.3748/ wjg.v16.i6.713

[13] TAN FL, OOI A, HUANG D, WONG JC, QIAN CN, et al. p38delta/MAPK13 as a diagnostic marker for cholangiocarcinoma and its involvement in cell motility and invasion. Int J Cancer. 2010; 126: 2353-61.

[14] BRIGGSA CD, NEAL CP, MANNA CD, STEWARD WP, MANSONA MM, et al. Prognostic molecular markers in cholangiocarcinoma: A systematic review. JEuropean of Cancer. 2009; 4: 33-47. http: //dx.doi.org/10.1016/j.ejca.2008.08.024

[15] MANCINO A, MANCINO MG, GLASER SS, ALPINI G, BOLOGNESE A, et al. Estrogens stimulate the proliferation of human cholangiocarcinoma by inducing the expression and secretion of vascular endothelial growth factor[J]. Dig Liver Dis. 2009; 41: 156-63. http: //dx.doi.org/10.1016/ j.dld.2008.02.015

[16] MONGIAT M, FU J, OLDERSHAW R, GREENHALGH R, GOWN AM, et al. Perlecan protein core interacts with extracellular matrix protein1 (ECM1), a glycoprotein involved in bone formation and angiogenesis. J Biol Chem. 2003; 278: 17491-99. http: //dx.doi.org/10.1074/jbc.M210529200

[17] FUJIMOTO N, TERLIZZI J, BRITTINGHAM R, FERTALA A, MCGRATH JA, et al. Extracellular matrix protein 1 interacts with the domain III of fibulin-1C and $1 \mathrm{D}$ variants through its central tandem repeat 2. Biochem Biophys Res Commun. 2005; 333: 1327-33. http: //dx.doi.org/10.1016/ j.bbrc.2005.06.046

[18] SERCU S, LAMBEIR AM, STEENACKERS E, EL GHALBZOURI A, GEENTJENS K, SASAKI T, et al. ECM1 interacts with fibulin-3 and the beta 3 chain of laminin 332 through its serum albumin subdomain-like 2 domain. Matrix Biol. 2009; 28: 160-169. http: //dx.doi.org/10.1016/ j.matbio.2009.02.003

[19] MCPHERSON L A, WOODFIELD G W, WEIGEL R J. AP2 transcription factors regulate expression of CRABPII in hormone responsive breast carcinoma. J Surg Res. 2007; 138: 71-78. http: //dx.doi.org/10.1016/j.jss.2006.07.002

[20] KENNY P A, ENVER T, ASHWORTH A. Receptor and secreted targets of Wnt- $1 / \beta$-catenin signalling in mouse mammary epithelial cells. BMC Cancer. 2005; 5: 3. http: //dx.doi. org/10.1186/1471-2407-5-3 